\title{
REFLEXIVE BANACH SPACES NOT ISOMORPHIC TO UNIFORMLY CONVEX SPACES ${ }^{1}$
}

\author{
MAHLON M. DAY ${ }^{2}$
}

Clarkson ${ }^{3}$ introduced the notion of uniform convexity of a Banach space: $B$ is uniformly convex if for each $\epsilon$ with $0<\epsilon \leqq 2$ there is a $\delta(\epsilon)>0$ such that whenever $\|b\|=\left\|b^{\prime}\right\|=1$ and $\left\|b-b^{\prime}\right\| \geqq \epsilon$, then $\left\|b+b^{\prime}\right\| \leqq 2(1-\delta(\epsilon))$. Milman ${ }^{4}$ and Pettis ${ }^{5}$ have demonstrated that any uniformly convex space is reflexive ${ }^{6}$ that is, that for each $\beta \in B^{* *}$ there is a $b \in B$ with $\beta(f)=f(b)$ for every $f \in B^{*}$. The same result clearly holds if $B$ is not uniformly convex but can be given a new norm defining the same topology under which the space is uniformly convex. It has been conjectured that every reflexive space can be given such a topologically equivalent uniformly convex norm; that is, that, in Banach's terminology, ${ }^{7}$ every reflexive space is isomorphic to a uniformly convex space. We shall show by a large class of examples that this is not the case; in fact the following result holds:

THEOREM 1. There exist Banach spaces which are separable, reflexive, and strictly convex, ${ }^{8}$ but are not isomorphic to any uniformly convex space.

We shall start with a class of Banach spaces and pick out a simple example having all but the strict convexity property; with this as a sample of what can happen we easily find a large number of spaces satisfying all the conditions of the theorem. As an application of our results we show that certain ergodic theorems of Alaoglu and Birkhof ${ }^{9}$ can be extended to some non-uniformly convex spaces.

\footnotetext{
${ }^{1}$ Presented to the Society, November 23, 1940, under the title Some more uniformly convex spaces.

${ }^{2}$ Corinna Borden Keen Research Fellow of Brown University.

${ }^{3} \mathrm{~J}$. A. Clarkson, Uniformly convex spaces, Transactions of this Society, vol. 40 (1936), pp. 396-414.

${ }^{4} \mathrm{D}$. Milman, On some criteria for the regularity of spaces of the type $(B)$, Comptes Rendus (Doklady) de l'Académie des Sciences de l'URSS, new series, vol. 20 (1938), pp. 243-246.

${ }^{5} \mathrm{~B}$. J. Pettis, $A$ proof that every uniformly convex space is reflexive, Duke Mathematical Journal, vol. 5 (1939), pp. 249-253.

${ }^{6}$ That is, "regular" in the terminology of Hahn. We use $B^{*}$ to denote the conjugate space of the Banach space $B ; B^{* *}=\left(B^{*}\right)^{*}$.

${ }^{7}$ S. Banach, Thêorie des Opérations Linéaires, Warsaw, 1932, chap. 11.

${ }^{8} B$ is strictly convex if the set of points with $\|b\|=1$ contains no line segments; that is, if $\|b\|=\left\|b^{\prime}\right\|=1$ and $b^{\prime \prime}=t b+(1-t) b^{\prime}$ with $0<t<1$, then $\left\|b^{\prime \prime}\right\|<1$.

${ }^{9}$ L. Alaoglu and G. Birkhoff, General ergodic theorems, Annals of Mathematics, (2), vol. 41 (1940), pp. 293-309.
} 
Let $\left\{B_{i}\right\}, i=1,2, \cdots$, be a sequence of Banach spaces and let $p$ be a real number $>1$. Let $B=\mathscr{P}^{p}\left\{B_{i}\right\}$ be the class of all those sequences $\boldsymbol{b}=\left\{b_{i}\right\}$ with $b_{i} \in B_{i}$ and $\|\boldsymbol{b}\|=\left(\sum_{i<\infty}\left\|b_{i}\right\| p\right)^{1 / p}<\infty$. It is easily verified that, with this norm and the obvious definitions of vector operations, $B$ is a Banach space.

LEMma. $\mathcal{P}^{p}\left\{B_{i}\right\}$ is reflexive (strictly convex) if and only if all $B_{i}$ are reflexive (strictly convex); in fact, $\left(\mathscr{P}^{p}\left\{B_{i}\right\}\right)^{*}=\mathcal{P}^{p^{\prime}}\left\{B_{i}^{*}\right\}$, where $1 / p+1 / p^{\prime}=1$.

For reflexivity we need only prove the last statement. If $\boldsymbol{f}$ is linear on $B=\mathscr{P}^{p}\left\{B_{i}\right\}$, let $\boldsymbol{b}_{i}$ be any element of $B$ of the form $(0,0, \cdots$, $\left.b_{i}, 0,0, \cdots\right)$ and define $f_{i} \in B_{i}^{*}$ by $f_{i}\left(b_{i}\right)=f\left(b_{i}\right)$; then there exist $b_{i} \in B_{i}$ with $\left\|b_{i}\right\|=1$ and $\left\|f_{i}\right\| / 2 \leqq f_{i}\left(b_{i}\right) \leqq\left\|f_{i}\right\|$ for all $i$. Since $\left\|b_{i}\right\|=1$ the sequence $\left\{\alpha_{i} b_{i}\right\}$ is in $\mathscr{P}^{p}\left\{B_{i}\right\}$ if and only if the sequence of real numbers $\left\{\alpha_{i}\right\}$ is in $l^{p}$. If $b^{j}=\left(\alpha_{1} b_{1}, \alpha_{2} b_{2}, \cdots, \alpha_{j} b_{j}, 0,0, \ldots\right)$ and $\boldsymbol{b}=\left\{\alpha_{i} b_{i}\right\} \in \mathcal{P}^{p}\left\{B_{i}\right\}$, then $\left\|\boldsymbol{b}^{j}-\boldsymbol{b}\right\| \rightarrow 0$ so $\boldsymbol{f}\left(\boldsymbol{b}^{j}\right) \rightarrow \boldsymbol{f}(\boldsymbol{b})$; clearly $\boldsymbol{f}\left(\boldsymbol{b}^{j}\right)$ $=\sum_{i \leqq j} \alpha_{i} f_{i}\left(b_{i}\right)$, so the series $\sum_{i<\infty} \alpha_{i} f_{i}\left(b_{i}\right)$ converges if $\sum\left|\alpha_{i}\right|^{p}<\infty$. Hence the sequence $\left\{f_{i}\left(b_{i}\right)\right\} \in l^{p^{\prime}}$ and therefore the sequence $\left\{\left\|f_{i}\right\|\right\} \in l^{p^{\prime}}$; so to each $f \in\left[\mathcal{P}^{p}\left\{B_{i}\right\}\right]^{*}$ we have associated a point $\left\{f_{i}\right\}$ of $\mathcal{P}^{p^{\prime}}\left\{B_{i}^{*}\right\}$ such that for any $\boldsymbol{b}=\left\{b_{i}\right\}, \boldsymbol{f}(\boldsymbol{b})=\sum_{i} f_{i}\left(b_{i}\right)$. Conversely if $\left\{f_{i}\right\} \in \mathcal{P}^{p^{\prime}}\left\{B_{i}^{*}\right\}$,

$$
\sum_{i<\infty}\left|f_{i}\left(b_{i}\right)\right| \leqq \sum_{i<\infty}\left\|f_{i}\right\| \cdot\left\|b_{i}\right\| \leqq\left(\sum_{i}\left\|f_{i}\right\|^{p^{\prime}}\right)^{1 / p^{\prime}}\left(\sum_{i}\left\|b_{i}\right\|^{p}\right)^{1 / p}
$$

by Hölder's inequality; so this sequence $\left\{f_{i}\right\}$ defines an $f \in\left(\mathscr{P}^{p}\left\{B_{i}\right\}\right)^{*}$, with $\boldsymbol{f}(\boldsymbol{b})=\sum_{i} f_{i}\left(b_{i}\right)$; clearly $\|\boldsymbol{f}\|=\left(\sum_{i}\left\|f_{i}\right\| p^{\prime}\right)^{1 / p^{\prime}}$, so we have completed our proof of reflexivity.

The proof of strict convexity is straightforward if we notice that strict convexity is equivalent to what Krein and $\breve{S}_{\text {mulian }}{ }^{10}$ call "strict normalization"; $B$ is strictly normalized if $\left\|b+b^{\prime}\right\|=\|b\|+\left\|b^{\prime}\right\|$ and $\left\|b^{\prime}\right\| \neq 0$ imply $b=t b^{\prime}, t \geqq 0$.

We now make a simple choice of the spaces $B_{i}$ to get an example of a reflexive space not isomorphic to a uniformly convex space. Let $B_{i}$ be the $i$-dimensional Minkowski space of points $b_{i}=\left(b_{i 1}, \cdots, b_{i i}\right)$ in which $\left\|b_{i}\right\|=\sup _{j \leqq i}\left|b_{i j}\right|$; then each $B_{i}$ is reflexive since it is finite dimensional, so $B=\Phi^{p}\left\{B_{i}\right\}$ is reflexive, by our lemma, for any choice of $1<p<\infty$. This $B$ can not be given a uniformly convex norm defining the same topology.

${ }_{10} \mathrm{~V}$. Smulian, On some geometrical properties of the unit sphere in the space of the type (B), Recueil Mathematique (i.e., Matematicheskii Sbornik), new series, vol. 6 (48) (1940), pp. 90-94. 
Suppose that there is such a norm-we shall represent it by $|b|$ defined in $B$. For $0<\epsilon \leqq 2$ we define $\delta(\epsilon)=\frac{1}{2}$ inf $\left(2-\left|b+b^{\prime}\right|\right)$, where the inf is taken over $b, b^{\prime} \in B$ with $|b| \leqq 1 \geqq\left|b^{\prime}\right|$ and $\left|b-b^{\prime}\right| \geqq \epsilon$; it can easily be shown that $\delta(\epsilon)>0$ for all such $\epsilon$ if and only if the norm $|b|$ is uniformly convex. For the topologies to be the same it is clear that there must exist $m, M$ with $0<m \leqq M<\infty$ such that $m\|b\| \leqq|b|$ $\leqq M\|b\|$; without loss of generality we can make $m=1$ by a change of scale of $|b|$ and get $\|b\| \leqq|b| \leqq M|| b||$ with $1 \leqq M<\infty$. If we fix any $i$ this must also hold in $B_{i}$ since $B_{i}$ is a closed linear subspace in $B$; so, if we let $K_{i}$ be the "sphere" of those points of $B_{i}$ with $\left|b_{i}\right| \leqq 1$, we see that $K_{i}$ is contained in the unit cube, $E_{b_{i}}\left[\sup _{j \leqq i}\left|b_{i j}\right| \leqq 1\right]$, and contains the cube $E_{b_{i}}\left[\sup _{j \leqq i}\left|b_{i j}\right| \leqq 1 / M\right]$. If we let $\alpha=1 / M$, we know that the points $\left(\epsilon_{1} \alpha, \epsilon_{2} \alpha, \cdots, \epsilon_{i} \alpha\right), \epsilon_{j}= \pm 1$, all lie in $K_{i}$. Considering, for example, $b=(\alpha, \cdots, \alpha, \alpha)$ and $b^{\prime}=(\alpha, \cdots, \alpha,-\alpha)$, we have $b-b^{\prime}=(0, \cdots, 0,2 \alpha)$, and $\left(b+b^{\prime}\right) / 2=(\alpha, \cdots, \alpha, 0)$. Now $\left|b-b^{\prime}\right|$ $\geqq|| b-b^{\prime} \|=2 \alpha$, so $\frac{1}{2}\left|b+b^{\prime}\right| \leqq 1-\delta(2 \alpha)$; that is, $|(\alpha, \cdots, \alpha, 0)| \leqq 1$ $-\delta(2 \alpha)$ or $|(\alpha /(1-\delta(2 \alpha)), \cdots, \alpha /(1-\delta(2 \alpha)), 0)| \leqq 1$. This could have been done with any choice of $\epsilon_{j}, j \leqq i-1$, so we can repeat $i-2$ more times, noting that $1-\delta(2 \alpha)<1$ and that $\delta(\epsilon)$ decreases as $\epsilon$ decreases, so that $\delta\left[\alpha /(1-\delta(2 \alpha))^{j}\right] \geqq \delta(2 \alpha), j=1, \cdots, i-1$; setting $b_{i}=\left(\alpha /(1-\delta(2 \alpha))^{i-1}, 0, \cdots, 0\right)$, we have $\left|b_{i}\right| \leqq 1$, but $\left|b_{i}\right| \geqq\left\|b_{i}\right\|>1$ if $i$ is large enough. This contradiction proves our theorem.

Since $B$ is clearly separable if all $B_{i}$ are, we could apply a theorem of Clarkson to show that an example satisfying all the conditions including strict convexity exists. However we need only take $B_{i}$ to have an $l^{q_{i}}$ norm instead of an $(m)$ norm and if $q_{i} \rightarrow \infty$ fast enough $\left(q_{i}=i\right.$ will do) then this new example is isomorphic to the old one while, by our lemma, it is strictly convex.

It is clear from the nature of our construction that if we had taken $B_{i}$ with the $l^{1}$ norm instead of the $(m)$ norm a similar contradiction would have appeared if we tried to fit in a uniformly convex norm (just for simplicity in the construction it is easier to take the dimension of $B_{i}$ to be $2^{i}$ in this case). Finally the finite dimensionality of the $B_{i}$ was used essentially only in proving reflexivity; this and the existence of subspaces of large dimension are our main requirements. Let $l_{\mu}^{q}, \mu$ a cardinal number $\geqq 0,1<q<\infty$, be the $\mu$-dimensional space with $l^{q}$ norm; in this notation the ordinary $l^{q}$ spaces would be $l_{\aleph_{0}}^{q}$. Using our example as a guide we have the following result:

THEOREM 2. The space $\mathcal{P}^{p}\left\{l_{\mu_{i}}^{q_{i}}\right\}$ is not isomorphic to a uniformly convex space, provided there exists a sequence $\left\{i_{j}\right\}$ of indices for which $\lim _{j} \mu_{i_{j}} \geqq \boldsymbol{N}_{0}$ while at the same time either $\lim _{j} q_{i_{j}}=+\infty$ or $\lim _{j} q_{i_{j}}=1$. 
In the first case we can show that $\mathscr{P}^{p}\left\{l_{\mu_{i}}^{q_{i}}\right\}$ has a subspace isomorphic to the example first given while in the second case it contains a subspace isomorphic to the example suggested in the paragraph preceding this theorem. By the same sort of embedding argument we can clearly get the same result for $\mathcal{P}^{p}\left\{L^{q i}\right\}$ if the $q_{i}$ are not bounded away from one and infinity.

A recent result of Boas is that $\Phi^{p}\left\{l^{q_{i}}\right\}$ is uniformly convex if $q_{i}=q$ for all $i$ and $1<q<\infty$. It is not yet known whether $\mathscr{P}^{p}\left\{l^{q_{i}}\right\}$ can be made uniformly convex, or is already so, if the $q_{i}$ are bounded away from 1 and $\infty$; i.e., if there exist $m, M$ such that $1<m \leqq q_{i} \leqq M<\infty$ for all $i$.

An application to ergodic theory. Milman and Alaoglu and Birkhoff have use for the following lemma:

If $K$ is a closed, convex, non-empty subset of a uniformly convex space, then there is a unique point in $K$ of least norm.

From a theorem of Mazur ${ }^{11}$ that any closed, convex set is closed under weak convergence we have the following result.

LEMMA. If $K$ is a closed convex non-empty set in a Banach space whose unit sphere is weakly compact, ${ }^{12}$ there is at least one point in $K$ of least norm. If $B$ is also strictly convex, then there is just one such point.

From this it follows that the general ergodic theorems depending on the minimal methods of proof of Alaoglu and Birkhoff hold in more spaces than the uniformly convex ones; we note the extensions of one or two of their theorems (we number them correspondingly); that the extensions are real and not merely formal follows from our existence theorem.

THEOREM 5. If $G$ is abelian, if $B$ is strictly convex and has unit sphere weakly compact, and if the norms of the operators $T_{g}$ are at most one. then every element of $B$ is ergodic.

THEOREM 6. If the norms of the operators $T_{g}$ are at most one, if the unit sphere in $B$ is weakly compact, strictly convex and without sharp edges, then every element of $B$ is ergodic.

${ }^{11} \mathrm{~S}$. Mazur, Über konvexe Mengen in linearen normierten Raümen, Studia Mathematica, vol. 4 (1933), pp. 70-84.

12 The unit sphere of $B$ is weakly compact if given any sequence $\left\{b_{n}\right\}$ of points with $\left\|b_{n}\right\| \leqq 1$ there is a subsequence $\left\{b_{n i}\right\}$ and a point $b_{0}$ (clearly of norm not greater than 1) for which $\lim _{i} f\left(b_{n_{i}}\right)=f\left(b_{0}\right)$ for all $f \in B^{*}$. 
Corollary. If $B$ is any $\mathscr{P}^{p}\left\{l_{\mu_{i}}^{q_{i}}\right\}$ or $\mathcal{P}^{p}\left\{L^{q_{i}}\right\}, 1<p<\infty, 1<q_{i}<\infty$, $\mu_{i}$ any cardinal $\geqq 0$, then every element of $B$ is ergodic.

Theorem 6 can be stated in a more symmetric form.

THEOREM $6^{\prime}$. If the norms of the operators $T_{g}$ are at most one, and if the unit spheres in both $B$ and $B^{*}$ are weakly compact and strictly convex, then every element of $B$ is ergodic.

By a theorem of Gantmakher and Smulian ${ }^{13}$ weak compactness of the unit sphere in both $B$ and $B^{*}$ follows from the weak compactness of either unit sphere; so this can be weakened slightly. Alaoglu and Birkhoff in a footnote remark that the condition that the unit sphere in $B$ have no sharp edges is equivalent to the condition that the unit sphere in $B^{*}$ be strictly convex. Note that a theorem of Milman asserts that a Banach space with a weakly compact and strictly convex unit sphere is reflexive.

Institute For ADVANCEd Study

${ }^{13} \mathrm{~V}$. Gantmakher and V. Šmulian, Sur les espaces linéaires dont la sphère unitaire est faiblement compact, Comptes Rendus (Doklady) de l'Académie des Sciences de l'URSS, new series, vol. 17 (1937), pp. 91-94. 